\title{
Acoustic Vector-Corrected Impedance Meter
}

\author{
Jonathan Scott, Senior Member, IEEE, and Kyle Pennington
}

\begin{abstract}
We describe the development of a novel instrument intended for the measurement of the acoustical reflection coefficient of materials. The instrument effectively implements a 1-port, vector-corrected, network analyzer in the acoustic, rather than the electromagnetic, domain. Employing the welldocumented methods of error correction familiar to microwave engineers, this instrument permits automated measurement of an acoustic impedance presented to a waveguide port. A dual directional coupler allows a working frequency range of well over an octave. In principle, a set of 6 couplers would allow measurement from 100 Hertz to 50,000 Hertz.
\end{abstract}

Index Terms-Acoustic measurements, acoustic impedance, acoustic reflection, impedance measurement, impedance tube, vector correction, network analyzer.

\section{INTRODUCTION}

The vector network analyzer is a familiar and powerful instrument that has been employed for many decades to obtain very accurate impedance measurements in high-frequency systems involving travelling waves and whose operating wavelengths range from tens of metres to fractions of millimetres [1]. Given good impedance standards against which to calibrate, it is capable of very good accuracy and superb resolution and repeatability [2]-[4]. Figure 1(a) shows the notional block diagram of a one-port Vector Network Analyzer (VNA) or Vector Impedance Meter (VIM). Such an instrument consists of a signal source, a mechanism for detection (separation) of the travelling waves carrying energy forward and backwards along a transmission line, an electronics package called a "vector receiver" that is able to measure the magnitude and phase of the travelling waves, and a load whose complex impedance is to be measured. The measurement yields an estimate of the ratio of incident and reflected waves, the reflection coefficient looking into the load, sometimes called the input scattering parameter

$$
S_{11}=\Gamma_{\mathrm{LOAD}}=\frac{b_{1}}{a_{1}}
$$

which is related to the impedance of the unknown load by

$$
Z_{\mathrm{LOAD}}=Z_{0} \frac{1+\Gamma_{\mathrm{LOAD}}}{1-\Gamma_{\mathrm{LOAD}}}
$$

where $Z_{0}$ is the characteristic impedance of the line connecting the measurement system to the load, $a_{1}$ is the incident travelling wave magnitude, and $b_{1}$ the reflected wave magnitude.

Figure 1(b) presents a block representation of a microwave impedance meter as it was routinely implemented by the

Jonathan Scott is with the School of Engineering, the University of Waikato, Hamilton, New Zealand. e-mail: jonathanscott@ieee.org

Kyle Pennington was with Gallagher Animal Management Systems, Hamilton, New Zealand.

Copyright (c) 2013 IEEE. Personal use of this material is permitted. However, permission to use this material for any other purposes must be obtained from the IEEE by sending a request to pubs-permissions@ieee.org.

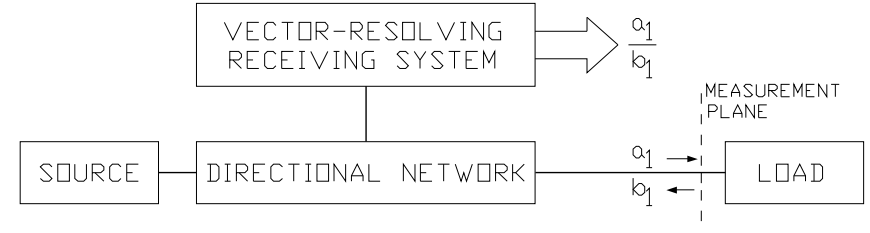

(a)

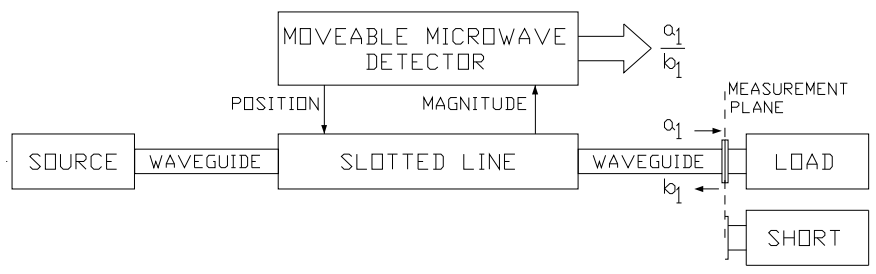

(b)

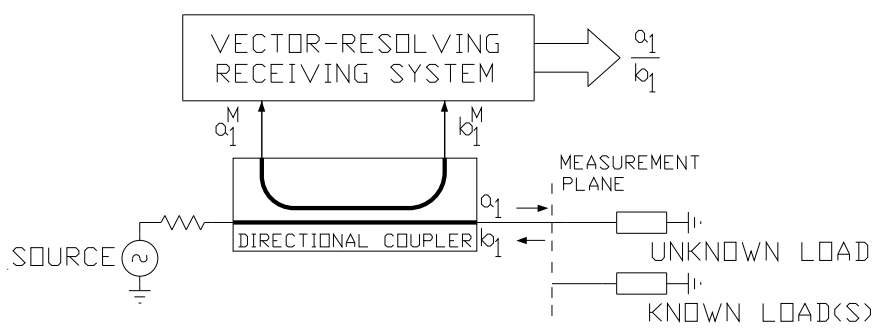

(c)

Fig. 1. (a) Notional form of a 1-port vector impedance measurement instrument. (b) Block diagram of an electromagnetic-waveguide impedance measurement system employing a slotted-line and a moveable detector. (c) Block diagram of a modern impedance measurement system using directional couplers.

1950s. Microwave signals can be conveyed in waveguide, and a slotted waveguide provides physical access to the standing wave pattern present in a waveguide transmission line as a result of a given load [5]. The measurement system needs only to measure position and magnitude of the standing wave in the guide. Phase calibration is achieved by temporarily replacing the load with a short (typically a cover plate on the guide flange) and noting the positions of the magnitude minima. The shift in position and depth of the minima, with the load in place of the short, allow the complex ratio of the forward and reverse travelling waves to be found.

Figure 1(c) shows the more familiar block diagram of a modern, swept-frequency, 1-port, network analyzer. In this instrument a reflectometer or directional coupler replaces the slotted line [1]. For reasons of convenience and bandwidth most VNAs use coaxial transmission lines below about $67 \mathrm{GHz}$. For high-power systems and for all systems above $110 \mathrm{GHz}$ waveguide is used. Imperfections in the receivers, directional networks, and interconnecting transmission lines mean that these systems must be calibrated through measurement of a set of known impedance standards. The calibration, 
referred to as vector correction, corrects for all imperfections. The process thus provides a path for traceability in the measurement, through the standards. In the case of a single-port impedance measurement, three known standards are required. The theory for such a calibration is presented in the Appendix.

\section{Acoustic Impedance MeAsurement}

Measurement of acoustic impedance is also of interest. The wavelengths of sound and ultrasound waves span the same range as that of electromagnetic signals from HighFrequency $(\mathrm{HF})$ to Millimetre-Wave $(\mathrm{mmW})$ as addressed by VNAs, and so the measurement challenges in the two domains have a lot in common. Various different approaches for acoustic impedance measurement have been reported in the literature. As in the case for electromagnetic (EM) energy, acoustic energy may be transmitted through a waveguide or "impedance tube". All reported methods employ some sort of waveguide [6]-[16]. ${ }^{1}$

The acoustic attenuator method essentially establishes an impedance divider in the guide [6]-[9]. Unfortunately, calibration of such systems leaves a lot to be desired. The most rigorous example is perhaps [8], for which the impedance reference is the calculated values of a series of closed tubes, and whose results rely on the source impedance of the measurement system. In [9] the source is contrived to offer a high characteristic impedance, reducing the dependence of the results upon its value. Rather than an impedance divider, the idea is to deliver a constant acoustic energy, analagous to measuring impedance with a current source. This is achieved with a capillary tube. A single closed tube is used for the calibration. The closedtube complex impedance, like those of [8], is calculated from theoretical expectations, a mechanism similar to the practice for development of standards in the microwave domain. This approach, where it can be implemented, is accurate provided the capilliary tube is of negligibly-high impedance. While it provides laudable results for the particular application in [9], the capilliary impedance is not accounted for in the calibration, and could affect measurements.

Measurement standards published for the guidance of acoustics companies use the standing-wave ratio (SWR) method [10], [11]. These methods are not exactly convenient, requiring a variety of delicate corrections, and in the end they are essentially equivalent to the slotted-line method of microwave fame, as they work back from the SWR in the guide without resolving the directional components explicitly [12]. The acoustic world is different from the electromagnetic in a number of ways. For example, it is not possible to construct a slotted waveguide as it is in the electromagnetic case, since any breach in the walls will radiate energy. One option is to move a microphone in the guide in some fashion that does not require an open breach in the tube, and trust that the mechanism causes a negligible disturbance, as is anticipated in [10].

\footnotetext{
${ }^{1}$ It should be noted that there are a number of reports in the literature of ingenious and well-developed acoustic measurements carried out in "free space", without benefit of a guide for the waves, see for example [17]. These must always present difficulty for traceable calibration, as it is not possible to reliably account for the dispersion of the transmission medium. This is the equivalent of attempting electromagnetic calibration in general in dispersive transmission lines such as coplanar waveguide or microstrip [18].
}

In theory, directional flows can be resolved in both electromagnetic and acoustic waveguides by sampling the magnitude of the standing wave at a number of fixed points. The familiar scalloped magnitude function is then fitted to the measured values, and the magnitudes of maxima and minima deduced once the function is found, as has been done in [13], [14]. After considerable investment of effort, our experience suggests that this method is less than reliable, especially in the presence of noise and measurement uncertainties, because of the need for a numerical fit [16].

An acoustic directional coupler developed using the theories originally used to design microwave waveguide couplers has been reported in [19]. We have constructed a number of such couplers, and assembled an acoustic instrument of the type represented in Figure 1(c). A photograph of a vector impedance meter assembled around one such coupler constructed in transparent acrylic material appears in figure 2 . A block diagram of the acoustic hardware appears in figure 3 . The waveguide inside dimension is $60 \mathrm{~mm} \times 60 \mathrm{~mm}$. The coupler-source assembly is made with $14 \mathrm{~mm}$-thick walls of clear acrylic. The offset loads are made with $5 \mathrm{~mm}$-thick acrylic walls. Each load-coupler junction consists of machined surfaces held in intimate contact by four, $4 \mathrm{~mm}$, stainless-steel bolts running through flanges on the loads and screwed into threaded holes tapped into the thicker coupler walls. Audio input and output of signals is achieved with 24-bit resolution using a Presonus AudioBox 1818VSL interfaced to a PC running MatLab. The microphones are Behringer ECM 8000 units. The source transducer is an 8-Ohm Visaton FRS 5 2.5inch driver. The expected working frequency of the coupler is nominally $1-2 \mathrm{kHz}$, but directivity drops off in the acoustic version less sharply than in the electromagnetic case, and we observed usable directivity from $750 \mathrm{~Hz}$ at the lower end to $2.25 \mathrm{kHz}$ at the upper end.

This class of instrument requires known standards for calibration. As in the case of the rectangular-pipe style of electromagnetic waveguide, there is no such thing as an "open" (infinite impedance) load, so calibrations such as the popular SOLT type routinely employed with coaxial transmission lines [4] are not possible. Nevertheless, full vector calibration can be carried out on 1-port electromagnetic waveguide systems, and this is the approach that we will explore in this manuscript.

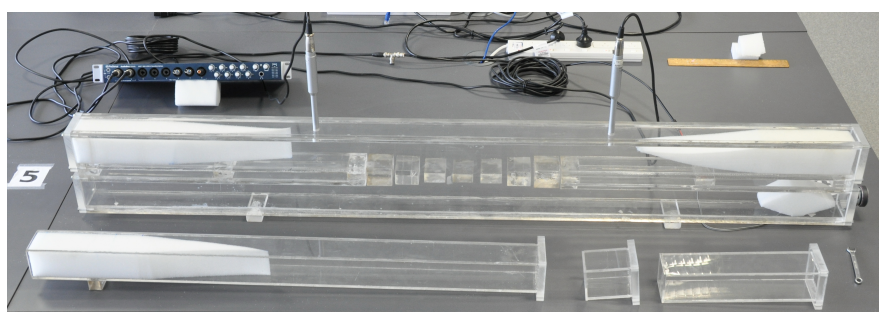

Fig. 2. Photograph of the directional coupler with a loudspeaker source and microphones attached. The source is at the right bottom of the coupler. The coupler has been constructed in transparent acrylic so that the parts are visible. The audio interface with 24-bit ADCs is shown at left rear. Two offset closed pipes and the sliding load are shown in front of the coupler. The ruler in the rear right of the image is $300 \mathrm{~mm}$ long to show scale.

Calibration of the instrument requires measurement of three 


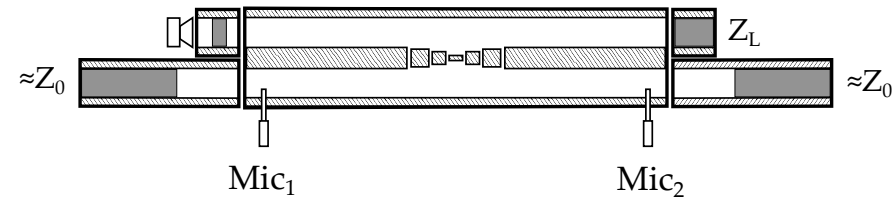

Fig. 3. Block diagram of the acoustic hardware. Microphones sense the sound pressure level in the two side arms of the coupler. The source is a small loudspeaker mounted behind an attenuating pad constructed of the same foam rubber used to make the loads.

known loads, as explained in the appendix. The simplest and most accurate load will be a "short", implemented as a sealed plate over the waveguide opening. In the electromagnetic domain the short standard has a reflection coefficient of unit magnitude and $\pi$ radians phase. An acoustic short has unit magnitude and zero phase. Provision of the other two known loads is much more complicated, and represents the greatest difficulty in realising an Acoustic Vector-corrected Impedance Meter (AVIM).

\section{SLIDING LOAD STANDARD}

An ideal load reflects none of the incident energy. If a load is to be used as a standard, the quality of calibration depends upon the extent to which it is possible to determine the $\Gamma_{\mathrm{LOAD}}^{M}$ that would result from signals $b_{1}^{M}$ and $a_{1}^{M}$ measured with such a perfect match presented at the measurement plane. If a load can be constructed whose residual reflection coefficient does not change with position along the waveguide, these desired measurements may be determined irrespective of the actual residual reflection coefficient with judicious choice of positions, using a mathematical process [20]. Consider the construction presented in figure 4. A realisation is visible at the front left of figure 2. Neglecting loss in the offset, and assuming the absorber is imperfect but moveable without change in a uniform guide, it will result in an impedance that describes a circle on the Smith Chart as the load moves along the waveguide. This occurs because the phase alone of the reflected energy is swept with position, and the sliding load would appear to a perfect meter as a circle whose centre lies at the centre of the Smith Chart. The centre of the circle actually measured represents the value that would be returned by the imperfect meter if the load were ideal.

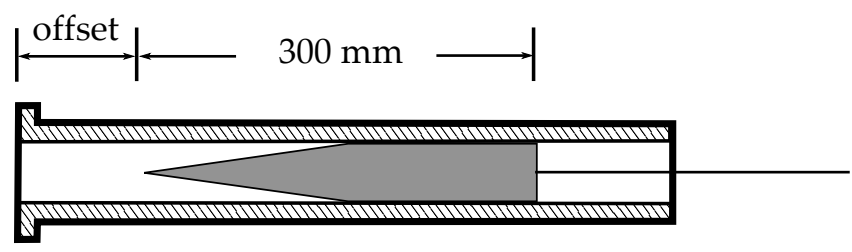

Fig. 4. A sliding load constructed as a wedge-shaped absorbing plunger.

The extent to which one can realise a load whose characteristics do not change as it is moved along a section of lossless waveguide is reflected in the extent to which the returned data represent a circle. The quality of the original load is reflected by the radius of the circle. The proximity of the circle's centre to the centre of the Smith Chart reflects the uncorrected quality of the analyzer hardware, essentially the raw directivity of the system. Thus measurement of a sliding load can say a lot about the quality of the hardware.

Figure 5 presents the result of measuring a sliding load at a number of positions. Note that the frequency range of the measurements extends beyond that over which the coupler is expected to work. The circles are all quite small. Over the expected working frequency range their centres give a reflection coefficient of less than $-20 \mathrm{~dB}$ before calibration. This shows that our coupler is giving good directivity. From the circle radii it can be seen that, at the majority of frequencies, the load has a return loss of $40 \mathrm{~dB}$ or more, and in all cases it is better than $20 \mathrm{~dB}$. This confirms that the load has adequately high return loss. It is also sufficiently invariant with position so that the circle model fits the data very well.

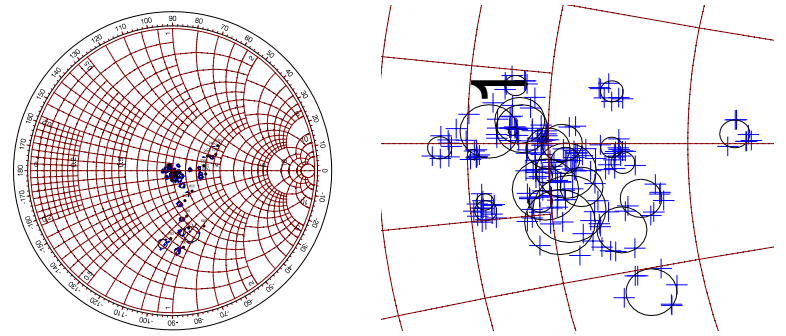

Fig. 5. Uncorrected measurements of the sliding load and the circles fitted to the data. Points nominally outside the usable frequency range of the coupler $(800-2200 \mathrm{~Hz})$ have been labelled with the measurement frequency. The righthand image is a zoom of the centre of the Smith Chart at left.

\section{OfFset ShORT STANDARdS}

Since an "open" standard does not exist in waveguide, socalled "offset shorts", in the form of lengths of waveguide sealed at the end, are used to realise the final impedance standards. In the acoustic case, more so than the electromagnetic case, loss in the offsetting line contributes to the perceived impedance of an offset short. In order to establish the impedance presented by an offset short, line loss must be found. We again resort to a sliding method, this time presenting a sliding reflection. The expected result of measuring a sliding reflection is again a circle on the Smith Chart if the line were to be lossless. In the case of a lossy offset transmission line, the action of sliding the reflector results in a spiral trajectory on the Smith Chart, as the length of line increases. The instantaneous radius of the spiral reduces as the length of lossy line increases. If the "slope" of the spiral-that is the rate at which its radius falls with plunger displacement — can be determined from such a measurement it becomes possible to calculate the impedance that is presented by a short of given offset, that is a closed pipe of known length. We fitted spirals to the data at each frequency using the algorithm of Taubin [21].

Figure 6 depicts two spirals plotted from the measured $\Gamma_{\mathrm{LOAD}}^{M}$ at representative frequencies and a number of positions 
of a substantially reflective plunger with known sequential displacements. The center of the spiral at each frequency and the rate of amplitude reduction with distance were found using [21]. Figure 7 shows some results after carrying out this procedure.

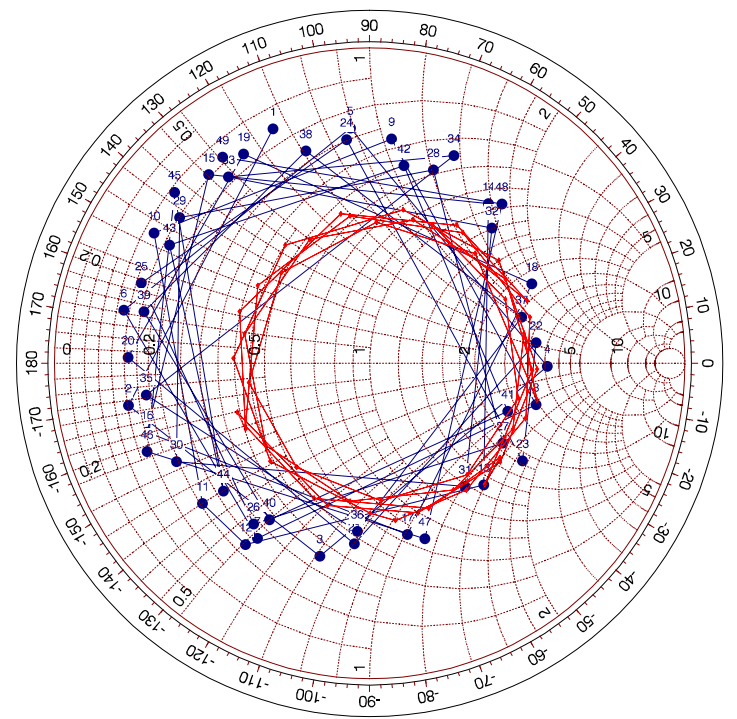

Fig. 6. Example spiral traces on the Smith Chart obtained by plotting measured, uncorrected, reflection coefficients at individual frequencies as a function of plunger position. Example spirals are shown at $1 \mathrm{kHz}$ and $2 \mathrm{kHz}$. The data at $2 \mathrm{kHz}$ is shown in blue with the data points numbered sequentially with plunger position. Data at $1 \mathrm{kHz}$ is red with diamond symbols.

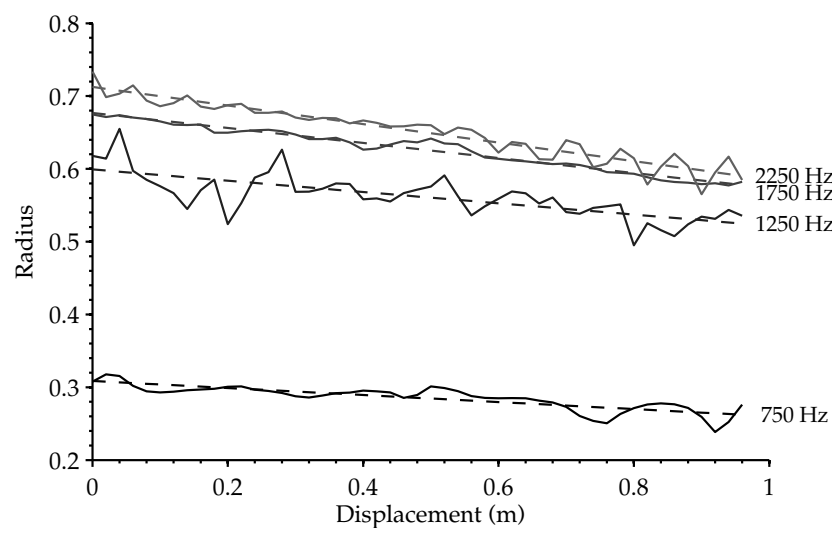

Fig. 7. Measured values of spiral radius against plunger displacement at some example frequencies (solid lines) compared to the values predicted from the fit (dashed lines).

Integrating the results, figure 8 shows the estimated loss in $\mathrm{dB}$ per meter as a function of frequency. Using this data, the expected impedances of the two offset shorts visible at the front in figure 2 were calculated.

Different offset loads have been characterised. The requirements for a robust calibration are that the three standard impedances used be spaced apart on the Smith Chart. Since we rely on a load, a short, and an offset short, the length of the offset needs to be distant from a multiple of half a wavelength, in order that the two impedance values whose

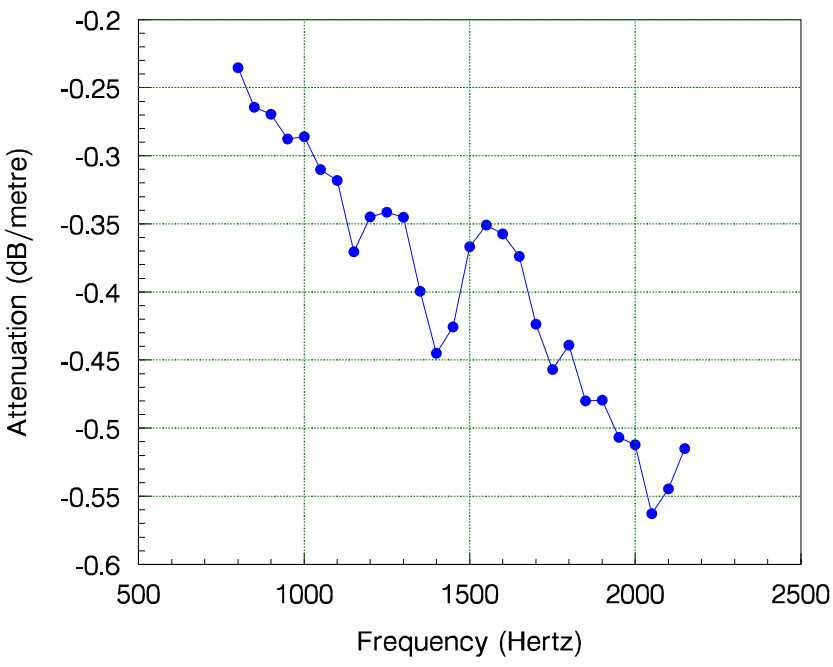

Fig. 8. Plot of the loss per metre of sound waves propagating through acrylic waveguide as a function of frequency found from linear fits to the estimates of spiral radii.

reflection coefficient approaches 1 do not coincide. It is usually considered that a spacing of at least $30^{\circ}$ on the chart is satisfactory. For the spread of frequencies here at least two shorts, in addition to the zero-length one, will be required.

\section{CONSISTENCY CHeCK}

At any single frequency the AVIM is calibrated using the plain short, the sliding load in a number of positions, and one or other of the offset shorts. The particular offset short is selected according to the relative proximity of the expected impedance to that of the plain short. Figure 9 shows the result of measuring the $300 \mathrm{~mm}$ offset short with the instrument once calibration has been carried out. Of course, the result is a perfect reproduction of its calculated impedance at frequencies where that standard is used as one of the three reference impedances. At other frequencies the errors of the instrument are evident. The errors after calibration appear to be in the region of \pm 1.0 to $1.5 \mathrm{~dB}$, which corresponds to a range of $12-19 \%$ of uncertainty in sound pressure level (SPL).

\section{Measurement of Soll and Pasture}

Our interest in this work is to characterise the acoustic "visibility" of pasture above soil. Our pasture sample was freshly-cut perennial ryegrass, averaging $20 \mathrm{~cm}$ in height, representative of what is found in paddocks and open land in dairy areas of New Zealand. It was transferred to the test waveguide promptly, preserving the soil integrity, and it was maintained in typical healthy conditions during the measurement. We repeated measurements to ensure that the sample was not degrading or materially changing. The entire process took only a few hours.

We first measured the reflection coefficient of the pasture, still rooted in a compacted soil sample, essentially a small square of soil with grass growing up from it extracted without disturbance. This was placed in the same open-ended length 

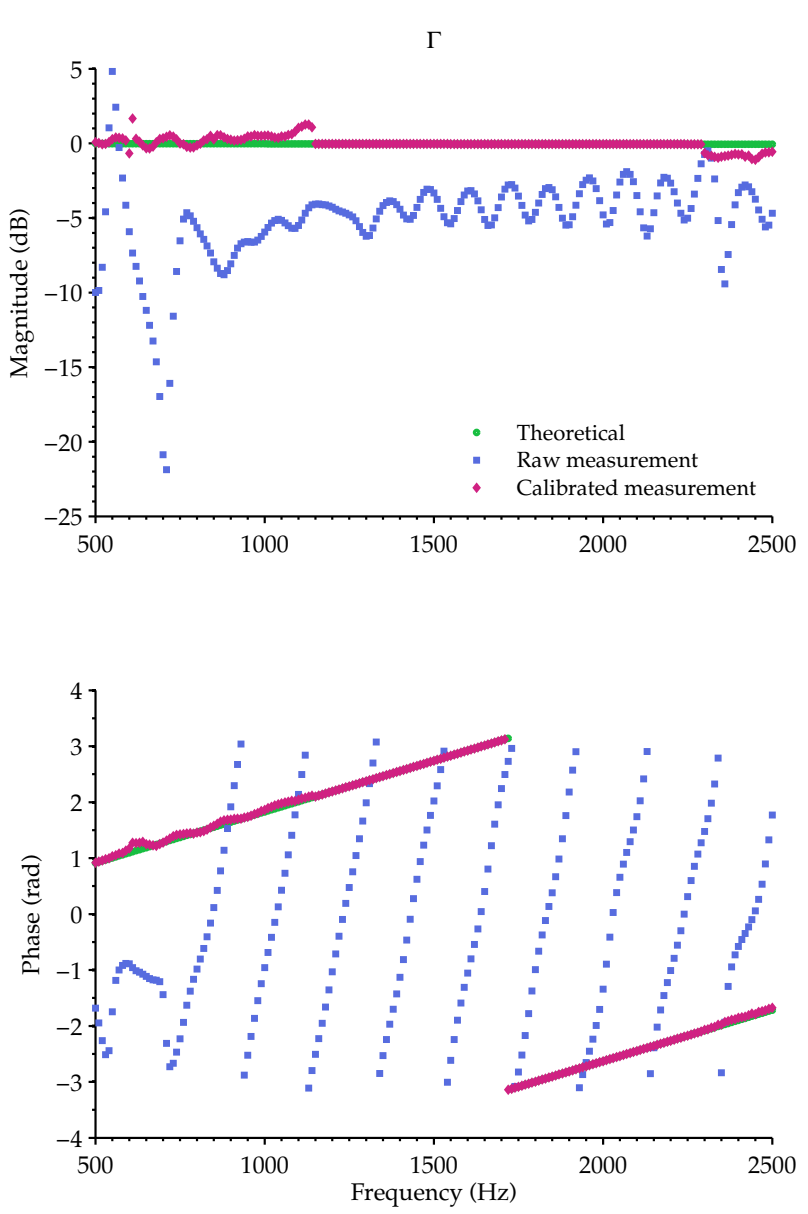

Fig. 9. Plot of the magnitude and phase of the first offset short. The regions where the calibration did not use this particular standard show the errors arising from the standard definitions we have used. Elsewhere the result is perfect to numerical precision by definition.

of transparent acryllic waveguide with $60 \mathrm{~mm} \times 60 \mathrm{~mm}$ cross section and the same $5 \mathrm{~mm}$ wall thickness used for the characterisation of offset loss as described above. We then measured the acoustic reflection coefficient of a sample of compacted soil by itself, and a sample of harvested pasture, both in the same sample guide. The results of these measurements are depicted in figure 10 .

We should ignore the results below about $800 \mathrm{~Hz}$ and above about $2200 \mathrm{~Hz}$ where coupler performance is falling away. In the usable range, the grass shows a reflection coefficient of $-20 \mathrm{~dB}$ or smaller. This indicates that in this frequency range the grass reflects very little energy, less than $1 \%$, with all of it being either absorbed or transmitted. We attribute the ripples visible at higher frequencies to interference with equally-small reflections from the environment behind the pasture material, an absorber in front of the open-end of the sample tube.

On the other hand, the compacted soil sample reflected about half of the incident power at $1 \mathrm{kHz}$. The fraction reflected fell steadily with frequency so that at $2 \mathrm{kHz}$ three-quarters of the power is absorbed and one-quarter reflected.

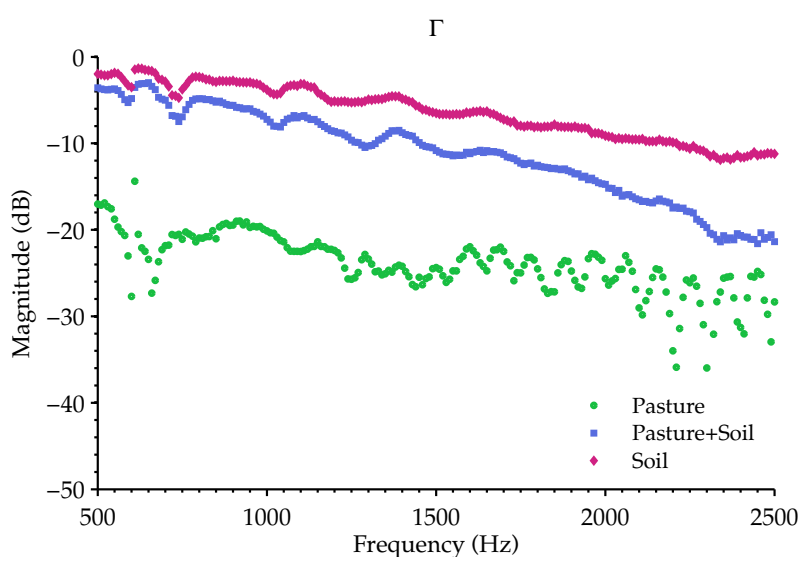

Fig. 10. Plot of the magnitude of $S_{11}$ with samples in an acoustic waveguide. The grass sample had the sliding load behind it when no soil was present. The presence of a load behind the soil sample had no effect.

When grass and soil are observed together, we see that 3 $6 \mathrm{~dB}$ less energy is returned than in the case of the soil alone. Since a relatively small fraction of energy is reflected from the grass, as evidenced from the grass-alone trace, we must conclude that the grass is absorbing a good deal of the energy, $1.5-3 \mathrm{~dB}$ on each pass (the $3-6 \mathrm{~dB}$ represents loss from a return trip through the grass). We may thus infer that

1) Soil reflects sound energy relatively well at low frequencies;

2) Soil reflects less energy at higher frequencies $(2 \mathrm{kHz}$ and above) than it does at low frequencies $(800 \mathrm{~Hz}$ and below);

3) Grass reflects little acoustic energy, about $1 \%$, with no obvious trend with frequency;

4) Grass absorbs energy, about $10 \mathrm{~dB} /$ meter at $1500 \mathrm{~Hz}$, with absorption increasing at higher frequencies.

These observations account for the inability of ultrasonicbased pasture meters to obtain a reliable measurement of the height of pasture. Rather, they achieve only a measurement of the distance to the top of the sward, since the echo from the ground is weak because of both the low reflection from the soil and the attenuation of the pasture.

\section{CONCLUSION}

We have constructed a vector impedance meter, essentially by realising the first 1-port vector network analyzer (VNA) in the acoustic rather than the electromagnetic domain. The directivity of the hardware is adequate for calibration over a frequency ratio of almost $3: 1$. We believe the accuracy to be around $\pm 1.5 \mathrm{~dB}$, limited by the availability of techniques and standards against which the instrument is calibrated. There remains much work to be done on this aspect of the instrument.

The electromagnetic VNA advanced over a number of decades to provide fast, convenient measurement of impedance with very high accuracy and traceability. We have already taken advantage of several of the advances applied to the EM version of VNAs to speed development of this acoustic version. The current instrument represents a level of sophistication roughly corresponding with that of the VNA of 3 
decades ago. This instrument promises to provide the same advantages in the acoustic realm as are afforded by the VNA in the EM domain. We believe it will prove to have application in architecture, sound reproduction, musical instrument design, biomedical diagnostics, and agriculture.

\section{ACKNOWLEDGEMENT}

This work was supported by Gallagher Animal Management Systems, the Ministry of Business, Innovation, and Employment, New Zealand, and the Faculty of Science and Engineering at the University of Waikato. The authors wish to express their thanks especially to Kerry Bodman and Tony Smith.

\section{APPENDIX}

\section{Calibration of A 1-PORT Vector Meter}

The waves actually measured by the receiving system in instruments of the type portrayed in figure 1(c) are $a_{1}^{M}$ and $b_{1}^{M}$. By definition we can write down equation (1) but the analyzer actually measures

$$
\Gamma_{\mathrm{LOAD}}^{M}=\frac{b_{1}^{M}}{a_{1}^{M}}
$$

where the measured signals $b_{1}^{M}$ and $a_{1}^{M}$ have been corrupted by the imperfections of the directional detection hardware. We seek a method of obtaining $\Gamma_{\text {LOAD }}$ given $\Gamma_{\text {LOAD }}^{M}$.

Using the well-known technique of modeling the circuit as an ideal VNA connected to the port through an error network [22], the flow diagram of figure 11 follows from the theory of signal graphs [23], [24] and some algebra yields the equation:

$$
\Gamma_{\mathrm{LOAD}}^{M}=I_{11}+\frac{I_{12} I_{21} \Gamma_{\mathrm{LOAD}}}{1-I_{22} \Gamma_{\mathrm{LOAD}}}
$$

which may be manipulated to give the correction equation:

$$
\Gamma_{\mathrm{LOAD}}=\frac{\Gamma_{\mathrm{LOAD}}^{M}-E_{d_{1}}}{E_{r_{1}}+E_{s_{1}}\left(\Gamma_{\mathrm{LOAD}}^{M}-E_{d_{1}}\right)}
$$

where the error terms $E_{d_{1}}=I_{11}, E_{s_{1}}=I_{22}$, and $E_{r_{1}}=$ $I_{12} I_{21}$ are the three error coefficients required at each calibration frequency. Their values (at each individual frequency) are determined by measuring three known terminations, typically two reflective and a resistive load. This determination of the error coefficients ("calibration") is accomplished by observing that equation 4 may be manipulated to become:

$$
\Gamma_{\mathrm{LOAD}}^{M}=E_{d_{1}}+\left(E_{r_{1}}-E_{s_{1}} E_{d_{1}}\right) \Gamma_{\mathrm{LOAD}}+E_{s_{1}} \Gamma_{\mathrm{LOAD}}^{M} \Gamma_{\mathrm{LOAD}}
$$

which, after a change of variable, has the form

$$
k_{1}=x+k_{2} y+k_{3} z
$$

where the $k_{i}$ are obtained from measurements of the known reflection coefficients, and $x, y$ and $z$ quickly yield $E_{d_{1}}=x$, $E_{s_{1}}=z$ and $E_{r_{1}}=y+x z$. After measuring three known loads the solution of three complex linear equations in three unknowns may be carried out with standard matrix arithmetic, giving values for the error coefficients. Thereafter equation (5) yields $\Gamma_{\text {LOAD }}$ given $\Gamma_{\text {LOAD }}^{M}$.

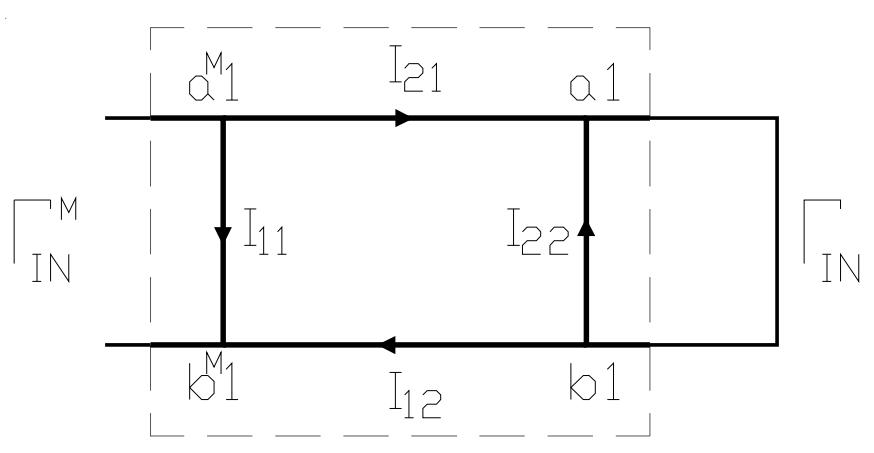

Fig. 11. Flow diagram for analysis of errors in a 1-port measurement network. The load impedance $\Gamma_{\mathrm{IN}}$ appears as $\Gamma_{\mathrm{IN}}^{M}$ as a result of the error network represented by the coefficients $I_{x y}$.

\section{REFERENCES}

[1] Rytting, Doug, "ARFTG 50 year network analyzer history", IEEE MTTS International Microwave Symposium Digest, 2008, pp11-18.

[2] Rytting, Douglas Kent "Network Analyzer Accuracy Overview", $58^{\text {th }}$ ARFTG Conference Digest, volume 40, Fall 2001, pp1-20.

[3] Marks, R.B., Jargon, J.A., and Rytting, D.K. "Accuracy of lumpedelement calibrations for four-sampler vector network analyzers", IEEE MTT-S International Microwave Symposium Digest, Volume: 3, 1998, pp1487-1490, 1998.

[4] Jargon, J.A., Marks, Roger B., and Rytting, D.K., "Robust SOLT and alternative calibrations for four-sampler vector network analyzers", IEEE Transactions on Microwave Theory and Techniques, Volume:47, Issue:10, 1999, pp2008-2013.

[5] Das, Annapurna, and Sisir Das, Microwave Engineering McGraw-Hill Education, 2009, ISBN 0-07-066738-1.

[6] Smith, J., Fritz, C. and Wolfe, J. "A new technique for the rapid measurement of the acoustic impedance of wind instruments", Proc. Seventh International Congress on Sound and Vibration, 4-7 July 2000, Garmisch-Partenkirchen, Germany, Vol III, pp.1833-1840.

[7] De Blok, C. M., and R. F. M. Van den Brink, "Direct-Reading One-Port Acoustic Network Analyzer", Journal of the Audio Engineering Society, vol. 41, no. 4, April 1993, pp231-238.

[8] D. H. Keefe, R. Ling, and J. C. Bulen, "Method to measure acoustic impedance and reflection coefficient," J. Acoust. Soc. Am., vol. 91, pp. 470-485, January 1992.

[9] Kausel, W., "Bore reconstruction of tubular ducts from its acoustic input impedance curve," IEEE Transactions on Instrumentation and Measurement, vol. 53, no. 4, August 2004, pp. 1097-1105.

[10] ISO 10534-1:1996, "Acoustics-determination of sound absorption coefficient and impedance in impedance tubes-Part 1: Method using standing wave ratio".

[11] ASTM Standard C384, 2004, "Standard test method for impedance and absorption of acoustical materials by impedance tube method".

[12] Clifton, Brian J. "Precision Slotted-Line Impedance Measurements Using Computer Simulation for Data Correction", IEEE Transactions on Instrumentation and Measurement, Volume: 19, Issue: 4, 1970, pp358364.

[13] William Johnston, "The Construction and Initial Measurements of an Acoustic Reflectometer", Internal report, Colorado State University, John Price Advisor. http://www.colorado.edu/physics/Web/reu/ Projects/Projects2007/WilliamJohnston.pdf See also http://spot.colorado. edu/ pricej/acoustics.html.

[14] John Price, "Acoustic VNA User's Guide", January 2008. http://spot. colorado.edu/ pricej/pdfdocs/AVNAUser' sGuide.pdf

[15] Peter D'Antonio, and Brian Rife, "The use of multi-microphone measurements of directional and random incidence acoustical coefficients", Journal of the Acoustical Society of America, April 2012; 131(4):3284.

[16] Kyle Pennington, Jonathan Scott, and Kerry Bodman, "Determining Acoustical Directionality in an Impedance Tube Using Multiple Fixed Microphones", Proceedings of the 18th ENZCon, Palmerston North, 2122 November, 2011, pp73-76.

[17] Chen, T., Ho Bong and H.R. Zapp, "Impedance and attenuation profile estimation of multilayered material from reflected ultrasound," IEEE 
Transactions on Instrumentation and Measurement, vol. 40, no. 4, August 1991, pp. 787-791.

[18] Jonathan Scott, "Investigation of a Method to Improve VNA Calibration in Planar Dispersive Media Through Adding an Asymmetrical Reciprocal Device", IEEE Transactions on Microwave Theory and Techniques, vol. 53, no. 9, September 2005, pp 3007-3013.

[19] Lagasse, P., "Realisation of an acoustical directional coupler", Journal of sound and vibration, 15(3), April 1971, pp367-372.

[20] H. C. Heyker, "The Choice of Sliding Load Positions to Improve Network Analyzer Calibration", 12th European Microwave Conference, Helsinki, September 1982, pp429-434.

[21] Taubin, Gabriel, "Estimation of planar curves, surfaces, and nonplanar space curves defined by implicit equations with applications to edge and range image segmentation," IEEE Transactions on Pattern Analysis and Machine Intelligence, vol. 13, no. 11, pp 1115-1138, November 1991.

[22] Rehnmark, Stig "On the Calibration Process of Automatic Network Analyzer Systems", IEEE Transactions onMicrowave Theory and Techniques, Volume:22, Issue:4, 1974, pp457-458.

[23] Mason and Zimmerman, Electronic Circuits, Signals \& Systems Wiley, 1960.

[24] Kuhn, "Signal Flow Graphs", Microwave Journal, November 1963, pp 59+.

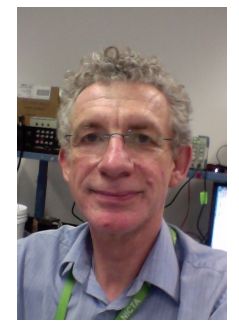

Jonathan Scott (M'80-SM'99) is the Foundation Professor in Electronic Engineering at the University of Waikato in Hamilton, New Zealand. From 1998 to 2006 he was with the Hewlett-Packard/Agilent Technologies, Microwave Technology Center in Santa Rosa, where he was responsible for advanced measurement systems. In 1998 he was Chief Engineer at RF Technology in Sydney. He was with The University of Sydney in the Department of Electrical Engineering prior to 1997. He is a Professorial Fellow of Macquarie University. Professor Scott has authored over 100 refereed publications, and he holds a number of patents.

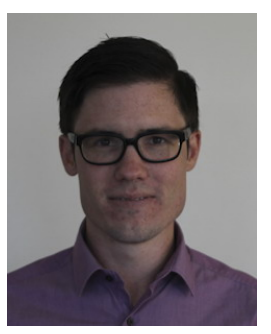

Kyle E. Pennington was born in Auckland, New Zealand, in 1986. He received the BE degree in electronic engineering from the University of Waikato in 2009. Since 2010, he has been working on completing his $\mathrm{PhD}$ in the field of acoustics. 